\title{
Jeunes Migrants Et Consommation De Drogues Dans La Region Du Haut-Sassandra En Cote d'Ivoire (Daloa)
}

\section{Ounnebo Gniondjibohoui Marc, Enseignant- chercheur}

UFR Sciences Sociales et Humaines, Département de SociologieAnthropologie, Université Jean Lorougnon Guédé, Daloa, Côte d'Ivoire

\section{Yao Kouakou Daniel, Enseignant- chercheur}

Université Jean Lorougnon Guédé-Daloa Côte d'Ivoire,

UFR Sciences Sociales et Humaines_ Groupe d'Etudes et de Recherches sur les Représentations Sociales (GERS_Abidjan)

François Yao Kouakou, Enseignant- chercheur

UFR Sciences Sociales et Humaines, Département de SociologieAnthropologie, Université Jean Lorougnon Guédé, Daloa, Côte d'Ivoire

Doi: 10.19044/esj.2017.v13n35p269 URL:http://dx.doi.org/10.19044/esj.2017.v13n35p269

\begin{abstract}
The purpose of the present work was to identify the explanatory factors of drug use among young migrants in the Upper Sassandra region, particularly in Daloa. It combined a mixed approach using a questionnaire and interview guide to collect data from all stakeholders involved in the relationship between young migrant status and drug use. In this perspective, young migrants as well as institutional and community actors were interviewed the geographical field of the sub-districts of the city of Daloa which is presented by several works as a hub of immigration. The working hypothesis that the practice of drug use among young migrants in Daloa is determined by factors related to social, psychological and financial vulnerability related to this status is validated in this work.
\end{abstract}

Keywords: Youth, Migrants, Drug Consumption

Résumé

Le présent travail avait pour objectif d'identifier les facteurs explicatifs de la pratique de consommation des drogues chez les jeunes migrants dans la région du Haut-Sassandra, notamment à Daloa. Il a conjugué une approche mixte en utilisant un questionnaire et un guide d'entretien afin de collecter les données auprès de tous les acteurs concernés par le lien qui existe entre statut de jeune migrant et consommation de 
drogues. Dans cette perspective, des jeunes migrants ainsi que des acteurs institutionnels et communautaires ont été interviewés le champ géographique des sous-quartiers de la ville de Daloa qui est présentée par plusieurs travaux comme une plaque tournante de l'immigration. L'hypothèse de travail qui soutient que la pratique de consommation de drogues chez les jeunes migrants à Daloa est déterminée par des facteurs liés à la vulnérabilité sociale, psychologique et financière en lien avec ce statut est validée dans le cadre de ce travail.

Mots-clés : Jeunes, Migrants, Consommation de Drogue

\section{Introduction}

La consommation des drogues ou substances psychoactives chez les jeunes en Afrique en général, et la Côte d'Ivoire en particulier constitue un problème majeur de santé publique. Ces conduites addictives les exposent à des risques sanitaire, sécuritaire et socioprofessionnel en termes de stigmatisations communautaires (Konaté, 2016 ; ONUCI, 2012). Les études conduites pour comprendre le processus d'adoption des pratiques de consommation des drogues chez les jeunes sont multiples et permettent d'identifier une pluralité de facteurs. Il s'agit à titre illustratif de facteurs liés au sexe, notamment les groupes de pairs déviants qui mésusent des substances psychotropes pour valoriser l'éthos masculin (Koudou, 2008; Lautier, 1987). Il convient également d'y ajouter la faiblesse de la répression institutionnelle, la structure familiale et singulièrement les familles monoparentales ou bien le statut d'orphelin, l'abandon parental et le parenting laxiste dans le domaine de l'éducation ou enfin, le facteur économique et financier à travers la variable pauvreté (Koudou, 2009). Cette diversité de facteurs interpelle les dimensions économiques, culturelles, sociales et psychologiques et ont pour point commun de mettre en avant, la vulnérabilité des jeunes qui versent dans ce type de conduite addictive (Nowlis, 1975 ; OMS, 2004).

Dans cette optique, le phénomène de la migration, autre préoccupation majeure en Afrique Subsaharienne, est de plus en plus associé à celui du trafic et de la consommation de la drogue chez les jeunes (Badie, 1993 ; Bredeloup, 1994). Ces deux thématiques polarisent le débat public dans la mesure où elles impliquent des acteurs adolescents qui sont victimes, le plus souvent, de réseaux mafieux qui exploitent leur fragilité psychosociale et leur désir de construire un avenir meilleur en Occident (Gravelaine et Senk, 1995 ; OMS, 1988).

Ainsi, le statut de migrant, en tant qu'il place l'individu, dans un environnement étranger, loin du réseau d'entraide communautaire, dans une situation de précarité financière, juridique, psychologique, voire linguistique, 
semble-t-il être un terreau fertile à l'adoption de pratiques de consommation de drogues (Mackay et Eriksen, 2002). Ce constat empirique, sous-tendu par certains travaux, tend à décrire ainsi la situation des jeunes migrations dans la région du Haut-Sassandra en Côte d'Ivoire. Ces travaux mentionnent la prégnance des facteurs liés à la pauvreté, la curiosité, la faiblesse de la réaction des forces de l'ordre et aux activités mafieuses de certains gangs dans la région Ouest de la Côte d'Ivoire (Konaté, 2016 ; REALIC, 2017). La ville de Daloa, en effet, est décrite comme une plaque tournante du trafic de drogues en plus d'être une zone de transit privilégié par les jeunes aussi bien nationaux qu'étrangers dans leur quête d'immigrer en Occident. Sur la route de cet Eldorado, nombreux sont ceux qui s'installent dans un " provisoiredéfinitif » à Daloa, du fait de l'addiction à la drogue. Face à ce problème social, les autorités politiques, administratives, les communautés ainsi que la société civile initient des actions récurrentes de sensibilisation, de prévention et de prise en charge des jeunes migrants. Pour autant, le phénomène des jeunes migrants, présentant des comportements addictifs face à la drogue, et alimentant la criminalité violente dans les milieux urbains en Afrique, demeurent une préoccupation pérenne (Coulibaly, Gregory et Piche, 1980 ; L'intelligent d'Abidjan, 2017).

Au-delà des invariants causals qui caractérisent le binôme drogueimmigration, il apparaît pertinent dans le présent travail, de s'intéresser au cas spécifique des jeunes migrants issus des pays limitrophes à la Côte d'Ivoire. La consommation de drogue, dans le parcours de ces jeunes migrants, se substitue à la quête d'un avenir meilleur en terre ivoirienne, de sorte qu'ils versent souvent dans la délinquance (Zanou et Dimbo, 1994). Le questionnement central de cette étude est le suivant : quels sont dès lors, les facteurs explicatifs de la consommation de drogues chez les jeunes migrants dans la région du Haut-Sassandra (Daloa)?

Sur la base de ces constats, l'objectif central de notre travail est d'identifier les facteurs explicatifs de la pratique de consommation des drogues chez les jeunes migrants dans la région du Haut-Sassandra, notamment à Daloa.

Nous pouvons retenir en hypothèse principale la pratique de consommation de drogues chez les jeunes migrants à Daloa est déterminée par des facteurs liés à la vulnérabilité sociale, psychologique et financière en lien avec ce statut.

\section{Methodologie}

\section{- Variables de l'étude}

La variable dépendante dans ce travail est le rapport à la drogue qui comprend deux (02) modalités, notamment la consommation de drogue et la non-consommation de drogues. Cette variable qualitative intègre au niveau 
de la modalité consommateur, une dichotomisation entre consommateur régulier et consommateur occasionnel.

La variable indépendante principale est liée au statut de migrant des jeunes enquêtés. Cette variable agrège des variables intermédiaires actives telles que l'âge, le niveau d'instruction, la nationalité des répondants, l'ancienneté du statut migratoire, raisons qui ont suscité cette migration et le statut socioprofessionnel (activité professionnelle).

\section{Terrain d'étude}

Notre terrain est la ville de Daloa qui est située au Centre-ouest de la Côte d'Ivoire. Elle comporte X sous-quartiers notamment Kennedy. La ville de Daloa située dans le Centre-Ouest de la Côte d'Ivoire à 400 kilomètres de la ville d'Abidjan et est le chef-lieu de la région du Haut-Sassandra depuis 1996. Daloa est limité au Nord par la ville de Vavoua et la ville de Zuénoula, au Sud par les villes d'Issia et de Sinfra, à l'Ouest par celle de Duékoué et Bangolo et à l'Est par la ville de Bouaflé. Daloa se trouve à la jonction de plusieurs axes routiers dont Abidjan-Guinée (Est-Ouest), San Pedro-Mali (Nord-Sud), Man-Abidjan et Odienné-San Pedro. Cette situation en fait un lieu de passage obligé, aussi bien pour le transport des marchandises que pour le transit de nombreux migrants qui fréquentent ces axes. Cette ville forestière à vocation agricole est un véritable centre de commerce et de transport. Elle comptait en 2014, 275.277 habitants et 42 quartiers pour une superficie de 5.305 hectares, ce qui correspondait à une densité de 52 habitants $/ \mathrm{km} 2$ (INS-RGPH, 2014). En conséquence, Daloa attire les populations des autres villes du pays et celles de la sous-région. La ville de Daloa est décrite par plusieurs études comme une plaque tournante de l'immigration aussi bien dans le pays que dans la sous-région ouest-africaine (Konaté, 2016) La figure (carte $\mathrm{n}^{\circ} 1$ ) localise et présente l'espace d'étude. 


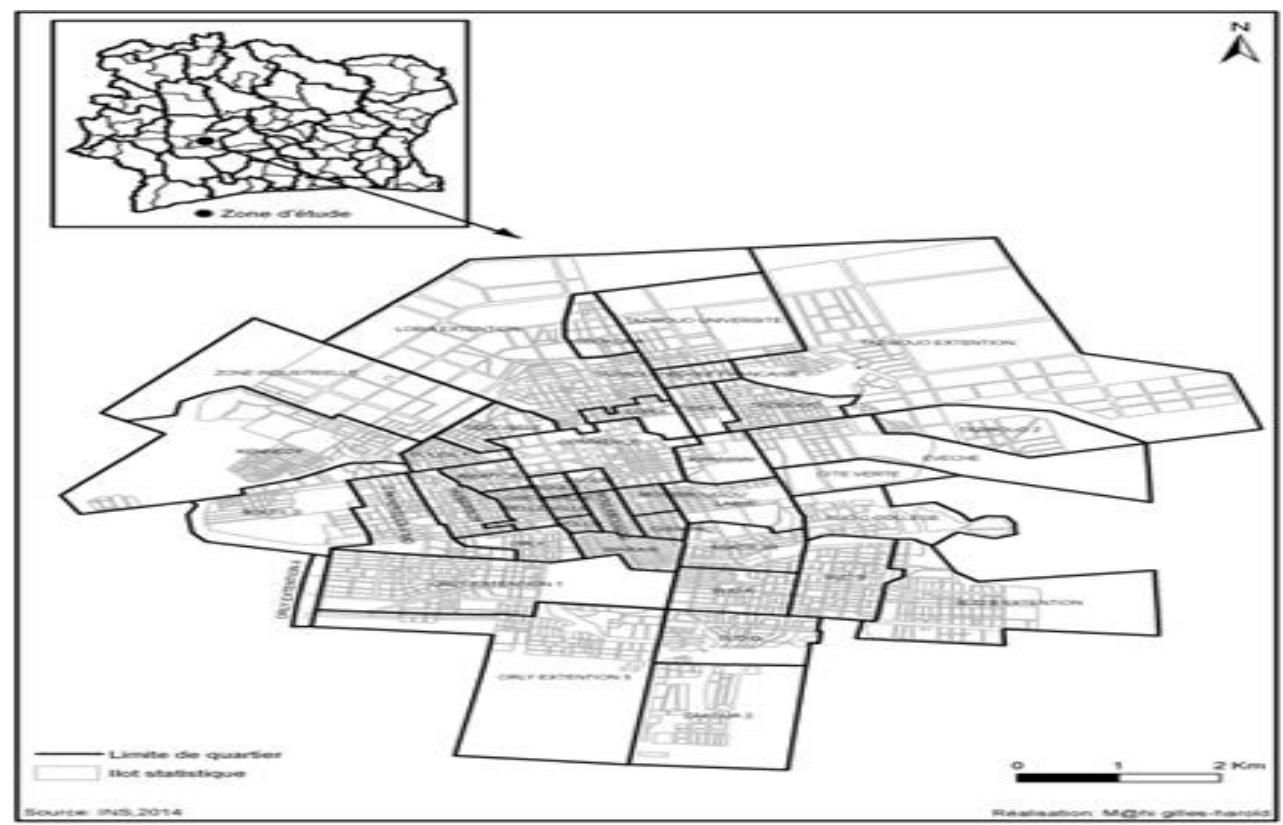

Figure 1 : Présentation cartographique des sous-quartiers de la ville de Daloa

L'étude s'est déroulée tout le long du mois de juin 2017 dans les différents sous-quartiers de la commune de Daloa.

\section{Population d'étude}

Le travail porte sur les jeunes migrants de la ville de Daloa. Ces enquêtés sont soit issus de l'immigration (première génération), soit ils sont nés de parents ayant immigrés en Côte d'Ivoire. En plus, ces répondants sont issus de plusieurs catégories socioprofessionnelles et résident dans les sousquartiers de la ville de Daloa. Ainsi, ces individus constituent un ensemble de répondants porteurs des informations relatives à la consommation de la drogue (consommateur et non-consommateur), en lien avec l'immigration.

L'étude intègre aussi les acteurs institutionnels (Agents de Police, Magistrats, Assistants sociaux, Educateur spécialisé, Responsables d'ONG de prise en charge des jeunes vulnérables à Daloa et Chefs communautaires). Toutes ces personnes ressources sont sollicitées afin de procéder à un recoupement des informations relatives au sujet étudié de sorte à comprendre davantage, les facteurs explicatifs de la persistance de ce phénomène malgré les efforts consentis par tous les acteurs pour son éradication (Aktouf, 1987).

\section{Échantillonnage}

La technique d'échantillonnage adoptée pour cette étude est l'échantillonnage accidentel. Nous avons néanmoins intégré le paramètre géographique dans la constitution du panel de répondant en investiguant sur 
les différents sous-quartiers de la ville de Daloa. Cette approche nonprobabiliste se justifie par la non-disponibilité des enquêtés qui œuvrent dans l'illégalité ainsi que le manque d'une base de données leur concernant sur le site (Grawitz, 2001).

Cette démarche nous a permis d'obtenir à la suite de la phase terrain, un total de 84 jeunes enquêtés au niveau des migrants. Ils ont un âge compris entre 15 et 18 ans dont $n=70$ individus de sexe masculin, soit $83 \%$ contre 13 individus de sexe féminin, soit, $16 \%$ de jeunes filles. Au niveau du niveau d'instruction, la majorité, $\mathrm{n}=60$, soit $71 \%$, pas eu accès à l'éducation conventionnelle. Ainsi, pour $n=39$, soit $46 \%$ d'entre eux ont fréquenté une classe de niveau secondaire contre $n=21$, soit $25 \%$ pour le niveau primaire tandis que $n=24$, soit $29 \%$ qui n'ont pas été scolarisés. Au niveau de la variable nationalité, ils proviennent des pays de la sous-région ouestafricaine notamment, le Burkina Faso $n=29$, soit, 35\%, la Guinée $n=17$, soit $20 \%$, le Mali $n=12$, soit $14 \%$, le Niger $n=12$, soit $14 \%$ et le Ghana $n=6$, soit $7 \%$. Nous avons enregistré $n=8$, soit $10 \%$ de non-réponses.

\section{Instruments et logiciel de traitement}

La méthodologie comporte un questionnaire destiné à identifier et comprendre le rapport des jeunes migrants à l'objet « drogue » à Daloa. Cet instrument explore ainsi, les grands axes liés au statut de migrant (nationalité, causes de l'immigration, statut des parents, occupation socioprofessionnelle, producteur, consommateur ou non de la drogue, projet d'avenir en termes d'abandon ou de persistance dans le lien addictif, rapport avec les forces de l'ordre, etc.). Le questionnaire est administré aux jeunes migrants uniquement et intègre des questions sur leur biographie (sexe, âge, niveau de scolarité, etc.).

Nous avons utilisé un guide d'entretien afin de cerner les opinions et perceptions des autres acteurs concernés par la thématique de l'immigration et de la drogue à Daloa (Agents de Police, Magistrats, Assistants sociaux, Educateur spécialisé, Responsables d'ONG de prise en charge des jeunes vulnérables à Daloa et Chefs communautaires). Le guide d'entretien reprend les grands axes identifiés au niveau du questionnaire en y incluant les politiques de prise en charge institutionnelle et communautaire de ces jeunes en situation de vulnérabilité psychosociale ainsi que les propositions de mesures pour optimiser ces initiatives d'assistance.

Le logiciel Sphinx Plus ${ }^{2}$ a été utilisé pour le traitement des données et les paramètres sociodémographiques en raison des fonctionnalités qu'il offre au niveau de l'analyse multivariée et lexicométrique pour les entretiens ouverts. 


\section{Resultats}

Typologie et Causes des migrations selon l'ancienneté migratoire à Daloa

Il s'agit ici, de déterminer la typologie des jeunes migrants selon le fait qu'ils sont nés en Côte d'Ivoire ou provenant des pays limitrophes.

Age $x$ Anciennetй migratoire a Daloa

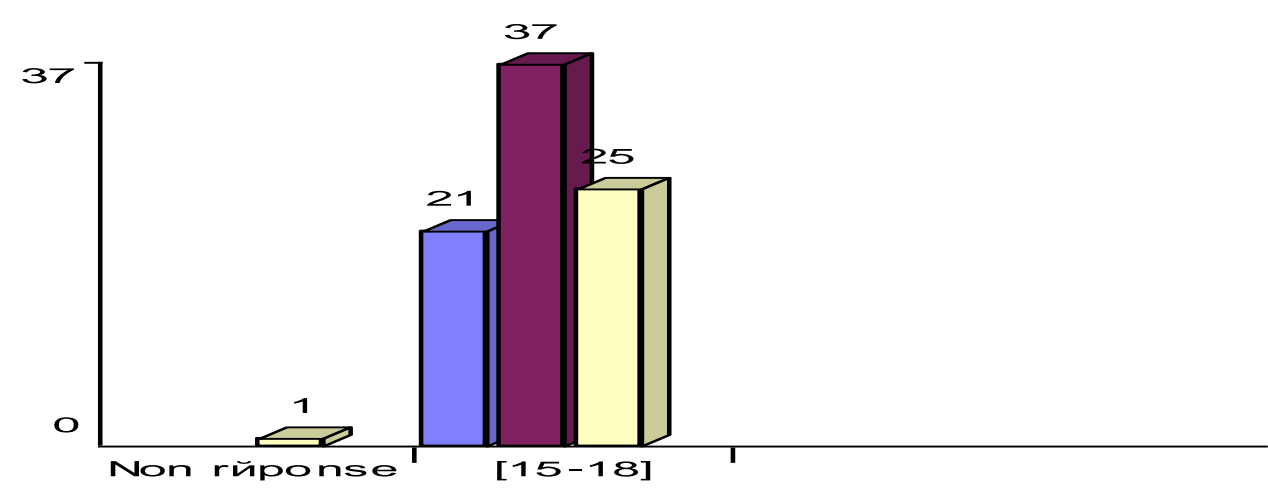

$\square$

Moins de 6 mo is

Depuis ma nais sance

$\square$ Depuis 1 an

Autre

Figure 2: Répartition des jeunes interviewés selon leur ancienneté migratoire à Daloa

La majorité des jeunes migrants proviennent des pays limitrophes avec $n=37$, soit $44 \%$ qui sont sur le site de l'étude depuis un an. Ensuite, $\mathrm{n}=25$, soit $30 \%$ soutiennent y être depuis leur naissance (migrants de seconde génération), contre $n=21$, soit $25 \%$ qui y ont migré depuis moins de 6 mois. Au total, la majorité $n=58$, soit $69 \%$ des jeunes interviewés est constituée de nouveaux migrants contre $21-25 \%$ d'anciens qui le sont par leur filiation.

\section{Raisons migratoires avancées par les jeunes}

Tableau 1: Répartition des jeunes interviewés selon les raisons migratoires

\begin{tabular}{ccc}
\hline Raisons de la migration & Nombre cité & Fréquence \\
\hline Non réponse & 24 & $\mathbf{2 8 , 6 \%}$ \\
\hline Trouver du travail & 24 & $\mathbf{2 8 , 6 \%}$ \\
\hline Rejoindre mes parents/amis & 29 & $\mathbf{3 4 , 5 \%}$ \\
Transition vers l'Europe & 9 & $\mathbf{1 0 , 7 \%}$ \\
Autre & 2 & $\mathbf{2 , 4 \%}$ \\
Total observé & $\mathbf{8 4}$ & $\mathbf{1 0 0} \%$ \\
\hline
\end{tabular}

La différence avec la répartition de référence est très significative. chi $2=30,07, \mathrm{ddl}=5,1-\mathrm{p}=>99,99 \%$. Le chi2 est calculé avec des effectifs 
théoriques égaux pour chaque modalité. Le nombre de citations est supérieur au nombre d'observations du fait de réponses multiples (4 au maximum).

La convergence considérable 58-69\% de ces jeunes migrants à Daloa dénote du fait que cette localité leur offre non seulement des opportunités économiques mais aussi, une plaque tournante déclarée depuis quelques années du réseau d'immigration clandestine vers l'Europe. Ainsi, le fait de rejoindre les parents ou amis 29-34,5\% est apparu comme un indicateur de premier plan pour expliquer leur présence à Daloa. Le second indicateur est celui de la quête d'emploi $24-28,6 \%$ contre une minorité $9-10,7 \%$ qui admet être en transit vers l'Europe. On note tout de même qu'une importante proportion 24-28,6\% ne s'est pas exprimée sur la question. A propos de l'immigration clandestine vers l'Europe, les propos recueillis auprès de certains acteurs institutionnels rendent bien compte de son ampleur à Daloa.

"L'immigration clandestine, c'est un phénomène typiquement propre à Daloa. C'est vrai que dans certaines régions il y a quelques-uns qui vont, mais à Daloa, les gens quittent les autres villes, d'autres pays pour prendre ici le départ. Daloa est donc devenu en quelques sorte, la plaque tournante de l'immigration clandestine »(OFK, Préfet de Police de Daloa).

«Les migrants ne sont pas que de jeunes ivoiriens. Ils sont de plusieurs nationalités. Ils se regroupent à Daloa car ils y bénéficient de réseaux bien organisés pour atteindre leur objectif. Celui d'avoir accès à l'Europe" (K. S., Substitut du procureur de Daloa).

\section{Profil psychosocial des jeunes migrants à Daloa}

\section{Activités professionnelles des jeunes migrants depuis 6 mois}

Tableau 2: Répartition des jeunes migrants selon leurs activités professionnelles

\begin{tabular}{ccc}
\hline Activité professionnelle depuis 6 mois & Nombre cité & Fréquence \\
\hline Rien/chômeur & 24 & $28,57 \%$ \\
\hline Commerçant & 28 & $33,3 \%$ \\
Syndicat/Transport & 9 & $10,7 \%$ \\
Cultivateur & 12 & $14,3 \%$ \\
Travailleur journalier & 11 & $13,1 \%$ \\
Total observé & 84 & $100 \%$ \\
\hline
\end{tabular}

La différence avec la répartition de référence est très significative. chi $2=48,48, \mathrm{ddl}=6,1-\mathrm{p}=>99,99 \%$. Le chi2 est calculé avec des effectifs 
théoriques égaux pour chaque modalité. Le nombre de citations est supérieur au nombre d'observations du fait de réponses multiples (6 au maximum).

La situation professionnelle des jeunes investigués depuis 6 mois, révèle que les jeunes migrants dans leur grande majorité $60-71,42 \%$, ils exercent dans le secteur informel. Cette donne implique des petits métiers notamment petit commerce 28-33,3\%, l'agriculture 12-14,3\%, travail journalier $11-13,1 \%$ et le transport 9-10,70\%. Cependant, $24-28,57 \%$ d'entre eux affirment être sans emploi. Ces petits métiers peu rémunérateurs et n'exigeant pas une main-d'œuvre qualifiée, constituent donc pour ces jeunes migrants en l'absence d'un emploi qualifié, des stratégies d'adaptation sociale.

\section{Activités professionnelles des Parents/Tuteurs des jeunes migrants}

A l'instar des enfants, les parents exercent dans leur ensemble dans le secteur informel. Ils sont majoritairement $31-36,9 \%$ de petits commerçants mais aussi travailleurs journaliers $30-35,7 \%$, transporteurs $19-22,6 \%$ et cultivateurs $15-17,9 \%$. A l'analyse de la situation socioéconomique de leurs parents, on peut affirmer que les jeunes migrants sont issus des creusets familiaux défaillants où leur intégration est parfois problématique. Leurs conditions de vie dépendent étroitement de celles de leurs parents. La rue dans ces conditions, est toute trouvée pour tenter l'aventure et se « débrouiller ». Dans un tel contexte de débrouille, apparaissent de nouvelles dynamiques non-conformistes d'adaptation sociale ou de survie comme la consommation et le trafic des stupéfiants.

Au sujet de la vulnérabilité socioéconomique qui caractérise les familles de ces jeunes migrants, les acteurs affirment ce qui suit :

« Depuis quelques années, les conflits sociopolitiques dans nos Etats africains ont provoqué la dégénérescence de la société dans tous les domaines: économique, familial, relationnel, social et professionnel de telle sorte que les familles sont déstructurées, en situation de précarité. Ces parents en raison de cette situation de précarité financière ne peuvent prendre correctement en charge leurs enfants. Ceux-ci se sentent obligés de tenter des aventures périlleuses, en quête de bonheur ou choisissent des activités illicites d'ascension sociale....".

(K.V., Educateur Spécialisé dans un centre social de Daloa). 
La migration du pays d'origine vers le pays d'accueil s'accompagne de profonds bouleversements de valeurs, d'identité et de statuts. Face au stress du chômage et de la pauvreté, aux difficultés de la vie quotidienne et à la dureté des activités de survie que la migration provoque, les jeunes migrants s'y adaptent d'une façon ou d'une autre. Ces bouleversements peuvent agir en synergie et développer des comportements addictifs.

\section{Rapport à la drogue chez les jeunes migrants à Daloa}

Tableau 3: Répartition des jeunes interviewés selon leur rapport avec la drogue

\begin{tabular}{ccc}
\hline Rapports à la drogue & Nombre cité & Fréquence \\
\hline Consommateur & 84 & $100 \%$ \\
\hline Producteur & 3 & $3,6 \%$ \\
Revendeur & 22 & $26,2 \%$ \\
Total observé & 84 & $100 \%$ \\
\hline
\end{tabular}

La différence avec la répartition de référence est très significative. chi $2=98,77, \mathrm{ddl}=3,1-\mathrm{p}=>99,99 \%$. Le chi2 est calculé avec des effectifs théoriques égaux pour chaque modalité. Le nombre de citations est supérieur au nombre d'observations du fait de réponses multiples (3 au maximum).

L'ensemble des jeunes migrants interrogés sont consommateurs de drogue contre $22-26,2 \%$ qui sont à la fois consommateurs-revendeurs et $3-3,6 \%$ qui sont à la fois consommateurs-producteurs.

«Le trafic et la consommation de la drogue ont pris des allures inquiétantes à Daloa ces dernières années. Qu'ils soient migrants ou non, toute la jeunesse est concernée...très souvent ils sont interpellés par la police... cela est bien dommage pour l'avenir de notre pays ». (S G., Sergent de Police à Daloa).

«Les statistiques relatives à l'interpellation des mineurs pour fait de drogue à Daloa depuis maintenant deux ans, placent les jeunes migrants en tête... Peut-être que cela est dî à leurs mauvaises conditions de vie...» (Sergent G. B., Direction des Drogues et Stupéfiants de Daloa). 


\section{Rapport à la drogue en fonction de la nationalité chez les jeunes migrants

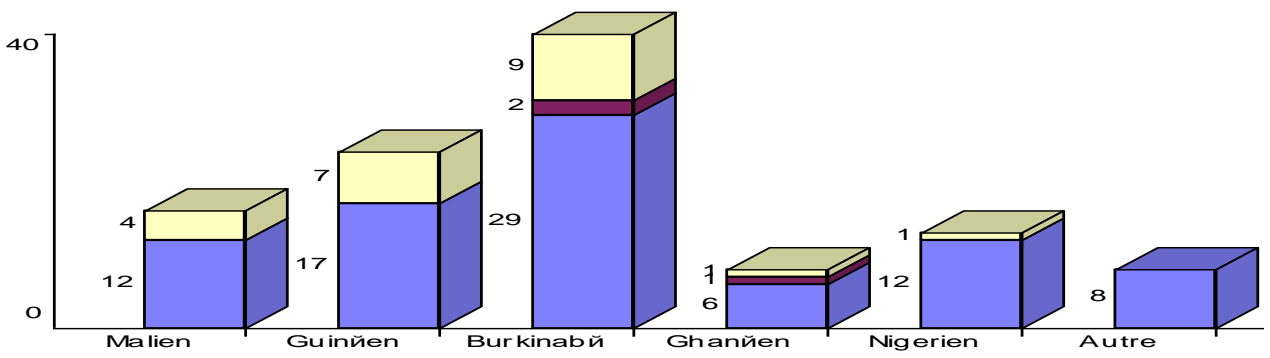

$\square$ Consommateur $\square$ Producteur $\square$ Revendeur

Figure 3 : Histogramme de répartition des jeunes migrants en fonction de la drogue et de la nationalité

Le facteur nationalité associé au rapport à la drogue révèle que les jeunes migrants burkinabés sont plus consommateurs de la drogue 29-35\% que leurs pairs de nationalités différentes. Outre la consommation, ils sont aussi revendeurs $9-11 \%$ et producteurs 2-2\%. Ensuite, viennent les jeunes migrants guinéens en matière de la consommation $17-20 \%$ et de la revente 7$8 \%$. Les jeunes maliens quant à eux viennent en troisième position de la consommation $12-14 \%$ et de la revente $4-5 \%$. De même que les jeunes nigériens au niveau de la consommation $12-14 \%$ mais un peu moins au niveau de la revente $1 \%$. Le dernier maillon est constitué de jeunes Ghanéens qui sont moins représentatifs aussi bien en matière de la consommation 6-7\% que de la revente $1 \%$ et la production $1 \%$.

Typologie de drogues et de consommateurs chez les jeunes migrants

Tableau 4: Répartition des jeunes interviewés selon la typologie des drogues consommées

\begin{tabular}{ccc}
\hline Type de drogues consommé & Nombre cité & Fréquence \\
\hline Cannabis & 82 & $97,6 \%$ \\
Cocaïne & 0 & $0,0 \%$ \\
Héroïne & 8 & $9,5 \%$ \\
Amphétamine & 37 & $44,0 \%$ \\
Autre & 1 & $1,2 \%$ \\
\hline Total observé & 84 & $100 \%$ \\
\hline
\end{tabular}

La différence avec la répartition de référence est très significative. chi $2=190,67, \mathrm{ddl}=5,1-\mathrm{p}=>99,99 \%$. Le chi2 est calculé avec des effectifs théoriques égaux pour chaque modalité. Le nombre de citations est supérieur au nombre d'observations du fait de réponses multiples (5 au maximum). 
Au niveau de la typologie des drogues consommées, l'enquête révèle que le cannabis est la drogue la plus consommées $82-97,6 \%$ par les jeunes migrants. Les amphétamines viennent en deuxième position $37-44 \%$ contre une minorité de consommateurs d'héroïne 8-9,5\%. Le taux élevé de la consommation du cannabis est dû au fait que le cannabis est une drogue cultivée localement. Il convient de relever, outre le cannabis, la consommation remarquable des amphétamines, produits issus du trafic international et qui constituent la deuxième classe de drogues la plus consommée dans le monde aujourd'hui et les drogues dures telle que 1'héroïne. Sur la base de ces données, le tableau $\mathrm{n}^{\circ} 4$ est présente une réalité préoccupante pour la localité de Daloa qui semble devenir une plaque tournante du trafic international des stupéfiants.

\section{Type de consommateur chez les jeunes migrants}

Tableau 5: Répartition des jeunes interviewés selon le type de consommateur

\begin{tabular}{ccc}
\hline Type de consommateur & Nombre cité & Fréquence \\
\hline Consommateur occasionnel & 60 & $71,4 \%$ \\
Consommateur régulier & 24 & $28,6 \%$ \\
Total observé & 84 & $100 \%$ \\
\hline
\end{tabular}

La différence avec la répartition de référence est très significative. chi $2=15,43$, ddl $=1,1-p=99,99 \%$. Le chi2 est calculé avec des effectifs théoriques égaux pour chaque modalité.

Du point de la fréquence, ils sont pour la plupart des consommateurs occasionnels $60-71,4 \%$ contre $24-28,6 \%$ de consommateurs réguliers. Qu'elle soit régulière ou occasionnelle, la consommation de la drogue entraine des risques évidents pour la santé intellectuelle, le bien-être physique et psychique du consommateur.

\section{Source d'approvisionnement de la drogue chez les jeunes migrants}

Tableau 6: Répartition des jeunes interviewés selon la provenance des drogues consommées

\begin{tabular}{|ccc|}
\hline Personne pourvoyeuse de la drogue & Nombre cité & Fréquence \\
\hline Non réponse & 7 & $8,3 \%$ \\
Amis & 56 & $66,7 \%$ \\
\hline Parents & 3 & $3,6 \%$ \\
\hline Corps habillés & 7 & $8,3 \%$ \\
Fumoir & 59 & $70,2 \%$ \\
Autre & 1 & $1,2 \%$ \\
\hline Total observé & 84 & $100 \%$ \\
\hline
\end{tabular}


La différence avec la répartition de référence est très significative. chi $2=170,38, \mathrm{ddl}=6,1-\mathrm{p}=>99,99 \%$. Le chi2 est calculé avec des effectifs théoriques égaux pour chaque modalité, type de drogues revendus.

Les jeunes migrants se procurent majoritairement les drogues consommées dans les fumoirs $59-70,2 \%$ et auprès de leurs amis $56-66,7 \%$ contre une minorité $7-8,3 \%$ qui affirme l'acquérir auprès des corps habillés. On note que l'offre de la drogue est une criminalité lucrative qui implique aussi les milieux criminogènes (fumoirs) que les personnes insoupçonnées tels les proches ou amis et les corps habillées.

" Le trafic de stupéfiants implique tout le monde... du petit chauffeur de taxi à la haute hiérarchie de notre société. Des individus fortunés, respectables sur qui ne pèse aucun soupçon, ils sont pour la plupart de grands hommes d'affaires, de grands hommes politiques ou d'Etat, des religieux influents...» (K. S., Substitut du Procureur de Daloa).

\section{Facteurs explicatifs (raisons de consommer la drogue)}

Les facteurs explicatifs sont l'ensemble des situations pré-criminelles qui conditionnent les jeunes migrants à la consommation des drogues. Ainsi quatre (4) raisons majeures sont identifiées (tableau ${ }^{\circ} 7$ )

Tableau 7: Répartition des jeunes interviewés selon les raisons de la consommation de la drogue

\begin{tabular}{|ccc|}
\hline Raisons de consommer la drogue & Nombre cité & Fréquence \\
\hline Manque de travail/oisiveté & 44 & $52,4 \%$ \\
\hline Coût de la drogue moins cher (accessible) & 33 & $39,3 \%$ \\
\hline Pour faire comme mes amis & 43 & $51,2 \%$ \\
\hline Pour me procurer du plaisir & 14 & $16,7 \%$ \\
\hline Pour supporter les difficultés de la vie quotidienne & 14 & $16,7 \%$ \\
\hline Pour supporter la frustration & 3 & $3,6 \%$ \\
\hline Pour vaincre la timidité & 22 & $26,2 \%$ \\
Pour oublier les soucis & 61 & $72,6 \%$ \\
\hline Pour avoir la force et travailler beaucoup & 20 & $23,8 \%$ \\
\hline autre & 22 & $26,2 \%$ \\
\hline
\end{tabular}

La différence avec la répartition de référence est très significative. chi $2=99,51, \mathrm{ddl}=10,1-\mathrm{p}=>99,99 \%$. Le chi2 est calculé avec des effectifs théoriques égaux pour chaque modalité. Le nombre de citations est supérieur au nombre d'observations du fait de réponses multiples (10 au maximum).

Il s'agit au niveau des facteurs de l'oubli des soucis $61-72,6 \%$ qui est apparu comme la première cause qui conditionne les jeunes migrants à la consommation des stupéfiants. La seconde raison évoquée est l'oisiveté 44- 
$52,4 \%$ suivie du mimétisme ou l'imitation pairs $43-51,2 \%$ et le faible cout de la drogue 33-39,3\% comme troisième et quatrième raisons. En plus de ces raisons majeures, d'autres facteurs notamment la timidité 22-26,2\%, le travail 20-23,8\%, la quête du plaisir 14-16,7\%, les difficultés de la vie quotidienne $14-16,7 \%$ et la frustration $3-3,6 \%$ sont entre autres raisons qui les poussent à la consommation des stupéfiants. On constate toute de même qu'une importante proportion $22-26,6 \%$ n'a pas émis d'opinion. Cela permet de conclure que les déterminants qui conditionnent ces jeunes migrants à la consommation des stupéfiants sont à la fois sociaux, psychologiques et économiques.

Les témoignages recueillis auprès des travailleurs sociaux qui interviennent dans la prise en charge psychosociales de ces jeunes présentant des addictions à la drogue nous permettent de fournir davantage d'informations sur la thématique :

" La défaillance des familles d'accueil et d'origines et les difficultés quotidiennes auxquelles ces jeunes sont confrontés développent en eux des sentiments d'exclusion, de frustrations, de vengeance... ce qui les poussent souvent à se réfugier derrière l'alcool et la drogue comme solutions...» (K.V., Educateur Spécialisé au centre social de Daloa).

"La jeunesse d'aujourd'hui, qu'elle soit migrante ou autochtone n'a pas de repères. La frustration du chômage et la pauvreté qui les caractérisent facilitent des comportements déviants... Ils ont besoins de la générosité de tous ». (Mlle. E.F., Chargé de Programme Protection de l'Enfance à la Clinique Juridique de Daloa).

Projet de désistement ou de persistance dans la consommation de drogue chez les jeunes migrants à Daloa

Tableau 8: Répartition des jeunes interviewés selon leur projet d'arrêter la drogue

\begin{tabular}{ccc}
\hline Projet d'arrêter ou non la consommation de drogue & Nombre cité & Fréquence \\
\hline Oui & 57 & $67,9 \%$ \\
Non & 27 & $32,1 \%$ \\
Total observé & 84 & $100 \%$ \\
\hline
\end{tabular}

La différence avec la répartition de référence est très significative. chi $2=10,71$, ddl $=1,1-p=99,89 \%$. Le chi2 est calculé avec des effectifs théoriques égaux pour chaque modalité.

$\mathrm{Au}$ niveau des perspectives en termes de projets ou intentions d'arrêter la consommation de la drogue, $57-67,9 \%$ des jeunes interrogés pensent pouvoir l'arrêter contre $27-32,1 \%$ qui admettent la poursuivre. 
Qu'ils aient l'intention ou non le projet d'arrêter la consommation de la drogue on constate que l'ensemble des jeunes migrants interrogés consomme la drogue. Or, la consommation d'un tel produit est de nature à affecter leur santé physique, mentale et psychologique. Ce qui conduira nécessairement à leur désintégration sociale.

\section{Discussion}

Le présent travail avait pour objectif général d'identifier les facteurs explicatifs de la pratique de consommation des drogues chez les jeunes migrants dans la région du Haut-Sassandra, notamment à Daloa. Il a conjugué une approche mixte en utilisant un questionnaire et un guide d'entretien afin de collecter les données auprès de tous les acteurs concernés par le lien qui existe entre statut de jeune migrant et consommation de drogues. Dans cette perspective, des jeunes migrants ainsi que des acteurs institutionnels et communautaires ont été interviewés le champ géographique des sous-quartiers de la ville de Daloa qui est présentée par plusieurs travaux comme une plaque tournante de l'immigration. Nous avions retenu en hypothèse de travail que la pratique de consommation de drogues chez les jeunes migrants à Daloa est déterminée par des facteurs liés à la vulnérabilité sociale, psychologique et financière en lien avec ce statut. Sur la base des résultats obtenus, nous pouvons soutenir que ces éléments valident l'hypothèse avancée dans le cadre de ce travail.

Ainsi, au niveau de la vulnérabilité sociale des jeunes migrants, nous pouvons noter que la majorité des jeunes migrants proviennent des pays limitrophes. La convergence de ces jeunes vers Daloa découle du fait que cette localité offre plusieurs opportunités économiques et migratoires car elle serait une plaque tournante déclarée depuis quelques années du réseau d'immigration clandestine vers l'Europe. Cette donne est contredite par les difficultés sociales que vivent ces jeunes dans la ville de Daloa car les parents et amis qui devraient servir de réseaux d'entraide communautaire sont très souvent démissionnaires (Blion, 1992). Par ailleurs, la situation sociale de ces jeunes migrants est caractérisée par la quête d'emploi pour une frange significative (Bredeloup, 1992 REALIC, 2017). Ils peinent à s'investir dans une formation professionnelle ou activité plus stable du fait de leur volonté d'aller «tenter leur chance »d'après leur rhétorique en Europe. Cette phase de transit vers l'Europe, les incite à faire de petits boulots, la plupart du temps dans le secteur informel, afin d'assurer les frais de l'immigration clandestine vers l'Europe. Tous ces éléments nourrissent une forme de vulnérabilité sociale qui les incite pour certains à consommer la drogue comme un élément d'exutoire (Abou, 1990 ; Konaté, 2016).

$\mathrm{Au}$ niveau de la vulnérabilité psychologique des jeunes migrants, nous pouvons noter que la situation professionnelle des jeunes investigués 
est celle de la précarité salariale faite de chômage et de petits métiers. Cette donne les place dans une situation de vulnérabilité psychologique. En plus, les parents de ces jeunes qui devraient les soutenir moralement ou psychologiquement sont eux-mêmes majoritairement confrontés à la même situation de fragilité psychologique du fait des incertitudes du lendemain liées à la situation socioéconomique. Ils éprouvent des difficultés d'intégration de sorte que les modèles familiaux sont défaillants et l'intégration est parfois problématique. Sur cette base, les rues de Daloa, dans ces conditions, deviennent le lieu de socialisation délinquante avec l'influence des groupes de pairs déviants, de consommation de drogues et d'adoption de comportements dérogatoires d'un point de vue pénal (Koudou, 2009 ; Mugenda, Mugenda et Olive, 2003). La logique de la « débrouille » dans l'aventure vers l'Europe est présente de manière récurrente chez les jeunes migrants qui apparaissent comme des consommateurs réguliers de la drogue selon les résultats de notre étude. Cette fragilité psychologique s'exprime également à travers l'incapacité qui les caractérise en ce qui concerne le projet de désistement ou de persistance dans la consommation de drogue (Koudou, 2008, 2009 ; Nkundimana, 2010). Ainsi, au niveau des perspectives, plus du tiers des répondants ne prévoient pas mettre fin à la consommation de la drogue. Dans l'ensemble, l'oubli des soucis apparait comme la première cause qui conditionne les jeunes migrants à la consommation des stupéfiants dans notre étude. En plus, la quête du plaisir, l'évacuation de la frustration et l'imitation des pairs délinquants sont d'autres raisons psychologiques avancés par les répondants (Born, 2005 ; Cusson, 1974a).

$\mathrm{Au}$ sujet de la vulnérabilité socioéconomique ou financière des jeunes migrants, il faut retenir qu'ils sont caractérisés par l'oisiveté, le chômage et la «débrouille » dans les activités précaires et informelles (Gonet, 1992 ; REALIC, 2017 ; Zanou et Dimbo, 1994). Le cumul de petits boulots, la plupart du temps dans le secteur informel, afin d'assurer les frais de l'immigration clandestine vers l'Europe, exprime la vulnérabilité financière de ces acteurs juvéniles en proie à la consommation des substances toxicomaniaques addictives à Daloa. Cette vulnérabilité financière est en lien avec la vulnérabilité sociale (Withol De Wenden, 1988 ; Zachariah, 1978) que nous avons décrite précédemment dans le cadre de la présente partie du travail.

\section{Conclusion}

La présente étude a permis de mettre en évidence, certains invariants causals en ce qui concerne l'étude de la consommation de la drogue (OMS, 2004). Il s'agit ainsi de la disponibilité ou facilité d'accès. $\mathrm{Au}$ niveau de l'environnement, nous retenons que les vulnérabilités des 
parents en termes de laxisme, de style d'éducation (trop sévère/trop tolérant) et les groupes de pairs déviants peuvent inciter à aller vers la drogue chez les jeunes (REALIC, 2017 ; Rolf, 1996). Le statut d'immigré constitue un facteur aggravant dans l'adoption des pratiques de consommation de drogues chez les jeunes dans la mesure où ces acteurs font face à des vulnérabilités multiples aussi bien au plan socioéconomique que psychologique. La drogue apparait comme une solution de désespoir face aux incertitudes du lendemain chez ces jeunes qui sont isolés socialement et psychologiquement dans des pays étrangers (Escohotado, 1995 ; François, 2002 ; Javier-Pérez 1990).

Face aux dangers multiples liés à la consommation des drogues, aussi bien au niveau de l'organisme (trouble mentale, hallucination, dépendance, probabilité de commission d'actes délinquants), il est nécessaire d'approfondir le lien entre les conduites addictives qui en découlent et le statut de migrant qui fragilise les jeunes qui vivent ces réalités en Afrique subsaharienne.

\section{References:}

1. Abou S., (1990). L'insertion des immigrés : approche conceptuelle, Les étrangers dans la ville, Éd. Simon-Barouh I. et al., Paris, L'Harmattan, 126-138.

2. Aktouf, O. (1987). Méthodologie des Sciences sociales et approche qualitative des organisations, Une introduction à la démarche classique et une critique, Les classiques des sciences sociales» [Site web : http://classiques.uqac.ca]

3. Badie, B., (1993). Flux migratoires et relations transnationales, Études Internationales, vol. XXIV, no 1, 7-16.

4. Blion R., (1992). Retour des Burkinabè de Côte d'Ivoire, Hommes et Migrations, no 1 160, décembre, 28-31.

5. Born, M. (2005). Psychologie de la Délinquance, De Boeck \& Larcier, Bruxelles.

6. Bredeloup, S., (1992). Itinéraires africains de migrants sénégalais, Hommes et Migrations, no 1 160, décembre, 16-22.

7. Coulibaly, S., Gregory J. et Piche V., (1980). Les migrations voltaïques: importance et ambivalence de la migration voltaïque, tome 1, INSD, CRDI, 411-422.

8. Cusson, M. (1974a). La resocialisation du jeune délinquant, Montréal, PUM. [Livre disponible dans Les Classiques des sciences sociales. JMT.].

9. Escohotado, A., (1995). Histoire élémentaire des drogues. Des origines à nos jours. Paris : Editions du Lézard. 
10. François, H., (2002). Les drogues et dépendances en 200 questions. Paris : Editions De Vecchi S.A

11. Gonet. L., (1992). Adolescents, drogues et toxicomanie. Collection « l'Essentiel ».

12. Gravelaine, de F. et Senk, P. (1995). Vivre sans drogues : Substances Toxiques, passions destructrices: l'expérience de ceux qui s'en sortent. Paris : Editions Robert Laffont, S.A.

13. Grawitz, M. (2001). Méthodes des Sciences Sociales, $11^{\text {ème }}$ éd. Dalloz, Paris.

14. Konaté, S., (2016). Samba Coulibaly appelle à une prise de conscience collective face au fléau de l'immigration clandestine. Daloa Info Bulletin, $\mathrm{N}^{\circ} 002$ / Octobre-Novembre-Décembre.

15. Koudou, O. (2008). Dysfonctionnements familiaux et formation de la personnalité à risque déviant chez l'adolescent. Revue Internationale de Criminologie et de Police Technique et Scientifique (RICPTS), Vol. LXI, n³, Genève, 259-272.

16. Koudou, O. (2009). «Développement et désistement du comportement délinquant chez l'adolescent ivoirien ». In Criminologie, vol. 42, $\mathrm{n}^{\circ}$ 1, 247-266. [URL http://www.erudit.org/apropos/utilisation.html].

17. L'intelligent d'Abidjan (2017). Daloa- Lutte contre l'immigration clandestine Une Ong lance le projet FAH-SO-KAFISSA. 07/04/2017, http://www.lintelligentdabidjan.info.

18. Lautier, F., (1987). En marge de la drogue: Toxicomanes dans les appartements Thérapeutiques. Paris : Les Editions ESF.

19. Mackay, J., Eriksen, M., (2002). The Tobacco atlas. Genève, Organisation Mondiale de la Santé.

20. Mugenda, G., et Mugenda, A. Olive. M., (2003). Research Méthodes. Nairobi - Kenya: Kenyatta University.

21. Nkundimana, J., (2010). Problématique de la consommation des drogues et ses conséquences sociales dans la Mairie de Bujumbura: cas du quartier Mutakura de la commune urbaine de Cibitoke. L'UEA. Licence, Burundi : Bujumbura.

22. Nowlis, H., (1975). La drogue démythifiée. Paris: La presse de l'UNESCO.

23. OMS (1988). Organisation Mondiale de la Santé/Conseil International des Infirmiers. Guide pour la prise en charge par les services infirmiers des porteurs du Virus de l'Immunodéficience Humaine (VIH), OMS SIDA Série 3.

24. ONUCI (2012). Trentième Rapport Périodique du Secrétaire Général sur l'Opération des Nations Unies en Côte d'Ivoire (S/2012/506).http://www.un.org/en/sc/International Organization. 
25. REALIC -Réseau ouest Africain de Lutte contre l'Immigration Clandestine- (2017). Daloa/Lutte contre l'immigration clandestine un Réseau ouest africain sensibilise les communautés en langues locales. Publié le 17 juillet 2017 | http://www. Abidjan.net.

26. Withol De Wenden, C., (1988). Les immigrés et la politique, FNSP, Paris.

27. Zachariah, K. C., (1978). La migration en Côte d'Ivoire, Banque Mondiale, Washington.

28. Zanou, B.C. et Dimbo T., (1994). Aspects démographiques et socioéconomiques des immigrations burkinabè vers la Côte-d'Ivoire, Karthala, Paris. 